\title{
Numerical Investigation on Spent Beam Deceleration Schemes for Depressed Collector of a High Power Gyrotron
}

\author{
Ioannis Gr. Pagonakis, Stefan Illy, Zisis C. Ioannidis, Tomasz Rzesnicki, \\ Konstantinos A. Avramidis, Gerd Gantenbein, Thorsten Kobarg, Bernhard Piosczyk, \\ Manfred Thumm, Fellow, IEEE and John Jelonnek, Fellow, IEEE
}

\begin{abstract}
Single stage depressed collector systems are widely used in high power gyrotrons in order to increase the overall efficiency. The position where the deceleration of the beam takes place influences the collector efficiency. In this work, several deceleration schemes are numerically investigated for the short pulse prototype of the $170 \mathrm{GHz}, 1 \mathrm{MW}$ European gyrotron for ITER. The results of this work are in a qualitative agreement with the results of dedicated experiments. A simple idea is also proposed for the significant increase of the overall gyrotron efficiency without an important technological modification.
\end{abstract}

\section{INTRODUCTION}

The idea of the application of a retarding voltage for the deceleration of the electron spent beam is widely used in high power gyrotrons for the increase of the overall efficiency for the last two decades [1]-[3]. The spent beam deceleration usually takes place either at the entrance of the electron beam in the mirror box (after the end of the quasi-optical launcher), as it is the case for the $140 \mathrm{GHz} 1 \mathrm{MW}$ gyrotron for Wendelstein W7-X [4] and the European (EU) $170 \mathrm{GHz}$, $1 \mathrm{MW}$ conventional cavity gyrotron for ITER [5], [6], or at the entrance of the beam in the collector, as it is the case for the $170 \mathrm{GHz}, 1 \mathrm{MW}$ Russian gyrotron for ITER [7], [8] and the European $170 \mathrm{GHz} 2 \mathrm{MW}$ coaxial cavity gyrotron [9], [10].

The position of the beam deceleration has a strong impact on the overall gyrotron efficiency due to the influence of the space charge voltage depression of the electron beam. Even if the second approach is more efficient for gyrotron operation, as it is presented in Sec. II, the first approach is more attractive for technological and practical reasons. Many manufacturing difficulties and gyrotron robustness issues are correlated to the large size of the fragile ceramic used in the second approach in which the isolation placed at the bottom of the large collector. In addition, the fact that a high

The authors are with the Institute for Pulsed Power and Microwave Technology, Karlsruhe Institute of Technology, 76131 Karlsruhe, Germany. email:ioannis.pagonakis@kit.edu

This work was supported in part by Fusion for Energy under Contracts F4EGRT-432 and F4E-GRT-553 to the European Gyrotron Consortium (EGYC). EGYC is a collaboration among CRPP, Switzerland; KIT, Germany; HELLAS, Greece; IFP-CNR, Italy. The views expressed in this publication do not necessarily reflect the views of F4E or the European Commission

The authors would like to warmly thank Dr. S. Alberti, Dr. J. Ph. Hogge, Dr. F. Albajar and Prof. I.L. Vomvoridis, for fruitful discussions, Dr. E. Borie and M. Schmid for their contribution to the text quality of the manuscript. Part of the simulations were performed at the CINECA super computer system at Milano, Italy. voltage is applied on the mirror box causes several additional complexities: (i) isolation is necessary between the gyrotron and the grounded magnet, (ii) a DC break is required at the connection between the window and the waveguide at the output, (iii) several difficulties occur at the installation and operation of a transverse sweeping system due to the fact that the coils are in close vicinity with high voltage components, (iv) the ion getter pumps are installed on the collector, further away from the magnetron injection gun (MIG), where a high quality vacuum is required. Furthermore, the position of the isolation ceramic at the bottom of the collector increases the risk of overheating from the stray radiation and a special cover must be used for its protection. Finally, the fact that a significant part of the gyrotron is at a high voltage in open air causes some security issues which is not the case in the first approach where all high voltage components are placed inside bore hole of the magnet which is usually filled with oil.

Due to all these reasons, the first approach for the spent beam deceleration was chosen for the collector of the EU gyrotron for ITER. Furthermore, an important design specification of that gyrotron was the technological similarity to the W7-X gyrotron in order to take advantage of the important experience gained on the specific technology during the many years of development of the series production tubes for the Wendelstein stellarator. Any discussion for modifications of the isolation ceramic position in the EU ITER gyrotron design would have caused an important technological modification which was out of the initial specifications and strategy.

In the context of the EU gyrotron development for ITER two prototypes have been manufactured: (i) the short pulse (SP) tube, manufactured at Karlsruhe Institute of Technology (KIT) [11] and (ii) the continuous wave $(\mathrm{CW})$ industrial gyrotron [12], manufactured by Thales Electron Device (TED). The SP prototype is a simplified version of the industrial prototype with the purpose to validate the design of the main gyrotron subcomponents, such as the electron gun, beam tunnel, cavity and quasi-optical output coupler with a pulse length up to $10 \mathrm{~ms}$. Oil cooling is applied at the electron gun similar to the industrial tube, while some water cooling is applied at the collector in order to increase the duty cycle. An important characteristic of the SP prototype is the modularity which provides us the possibility to easily exchange and test different subcomponents. The SP prototype was used in order to experimentally test the different deceleration schemes. 


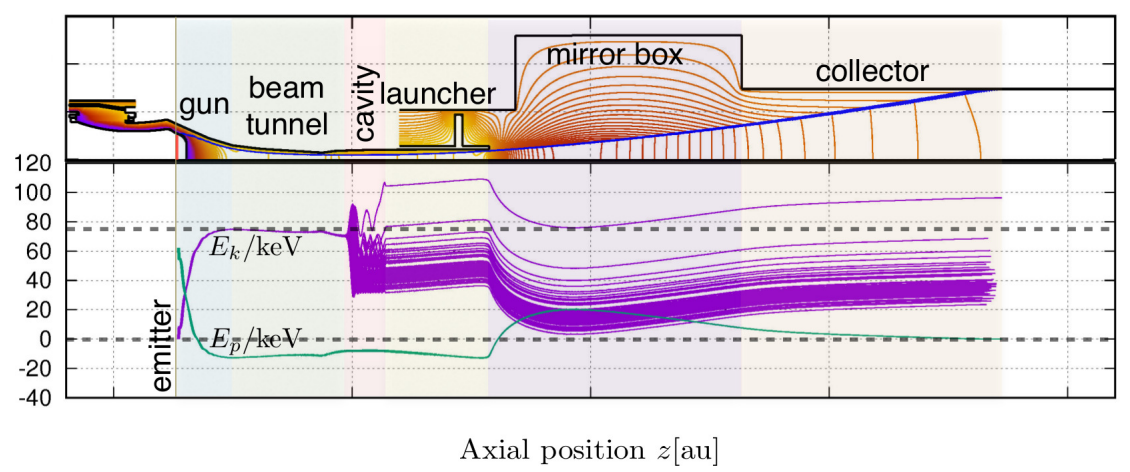

Fig. 1. Sketch of the SP prototype gyrotron shown at top while the kinetic $E_{k}$ and the potential $E_{p}$ energy of the beam electrons as a function of the axial position are plotted at the bottom.

A sketch of the SP prototype design, the electron beam and the equipotential lines are shown at the top of Fig. 1 while the kinetic and the potential energy of the electrons as a function of the their axial position are presented at the bottom of Fig. 1 as they are numerically calculated using the full gyrotron simulation approach [13]. The beam electrons are emitted from the heated emitter ring, are accelerated in the gun region and then are guided through the beam tunnel to the cavity, where the interaction with the nominal cavity mode takes place. The majority of the electrons lose part of their kinetic energy which is transformed to microwave energy. The beam electrons leave the cavity with a significant energy spread as shown in Fig. 1. Then, the kinetic energy of the electrons is slightly increased until the end of the launcher. A strong deceleration of the beam electrons takes place during their entrance in the mirror box due to the influence of the retarding voltage and the space charge voltage depression. Later, the kinetic energy of the beam electrons is significantly increased again while they are approaching the collector wall.

Using the electron optic code Ariadne [14] it was possible to calculate the influence of the nominal cavity mode on the beam electrons and to estimate the beam properties along the complete gyrotron geometry. The nominal radio-frequency (RF) field at the cavity was calculated with the cavity interaction code EURIDICE [15]. In order to keep the simulation time short, axisymmetric simulation takes place by choosing an azimuthal angle of the gyrotron geometry based on the worst case scenario concerning the collector efficiency. For the nominal SP prototype design, the azimuthal angle at which no mirror parts of the quasi-optical system are present is chosen.

Several deceleration schemes are investigated for the SP prototype gyrotron. Both approaches concerning the position of the isolation ceramic are theoretically and experimentally studied. The results of this analysis are presented in Sec. II. In Sec. III, a simple approach is proposed in which keeping the isolation ceramic at the bottom (first approach) and therefore maintain the technological advantages discussed earlier, keep the collector efficiency at a fairly high level. In Sec. IV, the main results of this work are briefly discussed.
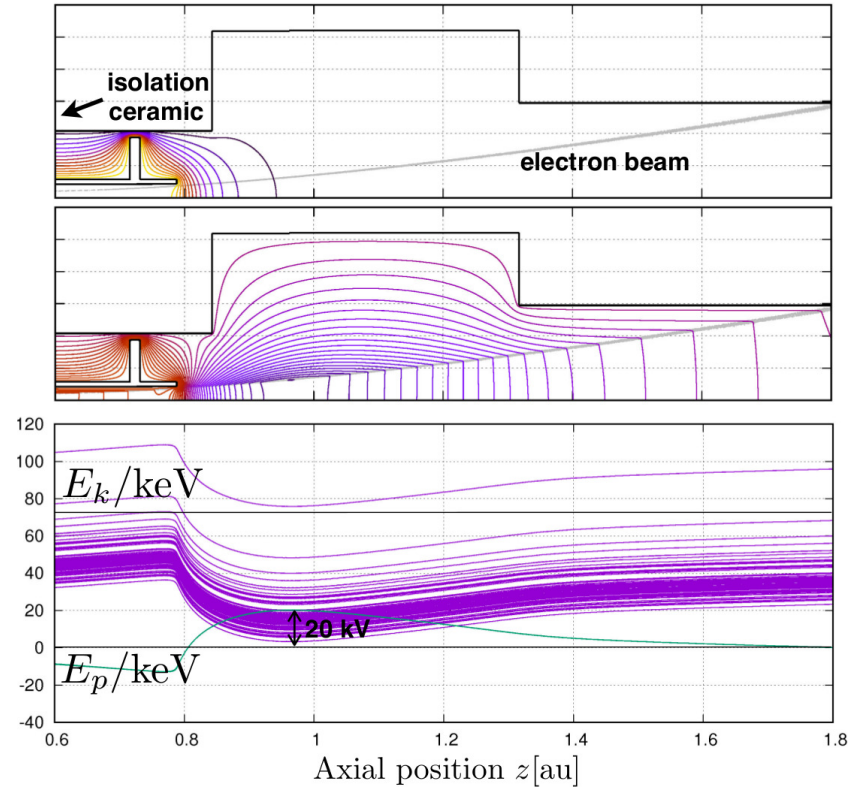

Fig. 2. The equipotential lines in the DS \#1 for $I_{b}=0 \mathrm{~A}$ and $I_{b}=45 \mathrm{~A}$ are plotted at the top and middle respectively, while the potential and the kinetic energy for a large number of sample beam electrons as a function of the axial position are presented at the bottom.

\section{Deceleration Schemes}

The first deceleration scheme (DS \#1) which has been investigated is the nominal design of the SP prototype gyrotron. In Fig. 2, the end of the launcher, the mirror box and the bottom part of the collector geometry are shown. The applied retarding voltage causes a deceleration of the electron beam at the entrance of the mirror box as is shown by the equipotential lines in the cases where the influence of the space charge of the spent beam is not taken into account $\left(I_{b}=0 \mathrm{~A}\right)$. The shape of the equipotential lines is dramatically modified under the influence of the beam space charge. The electron beam energy is strongly influenced by the additional deceleration at the entrance of the mirror box due to the space charge voltage depression. Then, the spent beam electrons are accelerated while they are approaching the grounded collector wall. The retarding voltage value was defined based on the criterion 


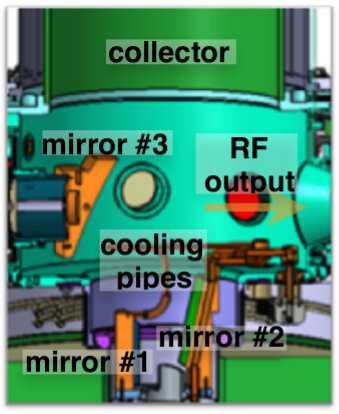

a. $C W$ prototype

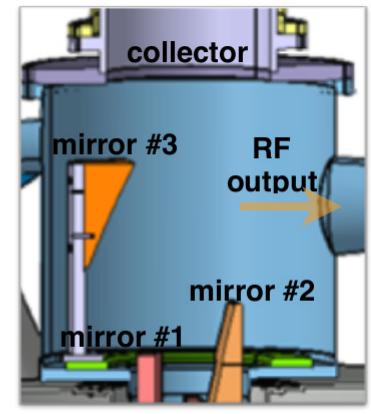

b. SP prototype
Fig. 3. Comparison of the $\mathrm{CW}$ with the SP prototype gyrotrons in the mirror box region.

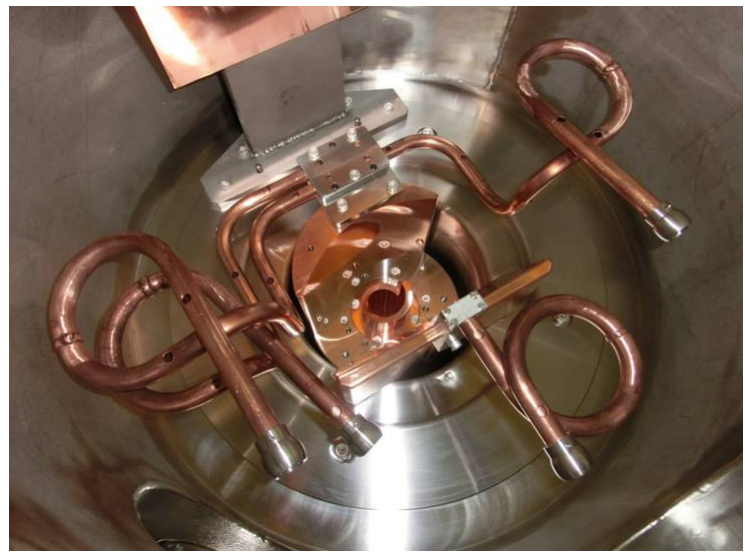

Fig. 4. Dummy pipes installed in the SP prototype to emulate the cooling pipes in the industrial prototype.

that no spent beam electron is reflected back into the cavity. According to the simulation, the maximum retarding voltage is limited to only $14 \mathrm{kV}$. It is remarkable that the kinetic energy gain of electrons from the middle of the mirror box towards the collector is at the level of $20 \mathrm{keV}$. This significant amount of energy is transformed to heat on the collector walls. The theoretical overall efficiency of the gyrotron using this deceleration scheme is about $36 \%$.

According to the simulation, the presence of the cooling pipes at the mirror box of the industrial prototype, as shown in Fig. 3, contributes to a small increase of the efficiency. In order to investigate that experimentally in the SP prototype tube, a complex system of pipes, shown in Fig. 4, has been designed and manufactured in order to emulate the cooling pipes in the $\mathrm{CW}$ prototype. The numerical simulation results of this deceleration scheme (DS \#2) are presented in Fig. 5. The presence of the cooling pipe at the anode voltage significantly modifies the shape of the equipotential lines and improves the operation of the single stage collector. The optimal applied retarding voltage is $23 \mathrm{kV}$, while the overall gyrotron efficiency increases to $42 \%$.

The third deceleration scheme (DS \#3) concerns the replacement of the nominal SP prototype collector with the collector of the modular SP coaxial cavity gyrotron [10] in which the
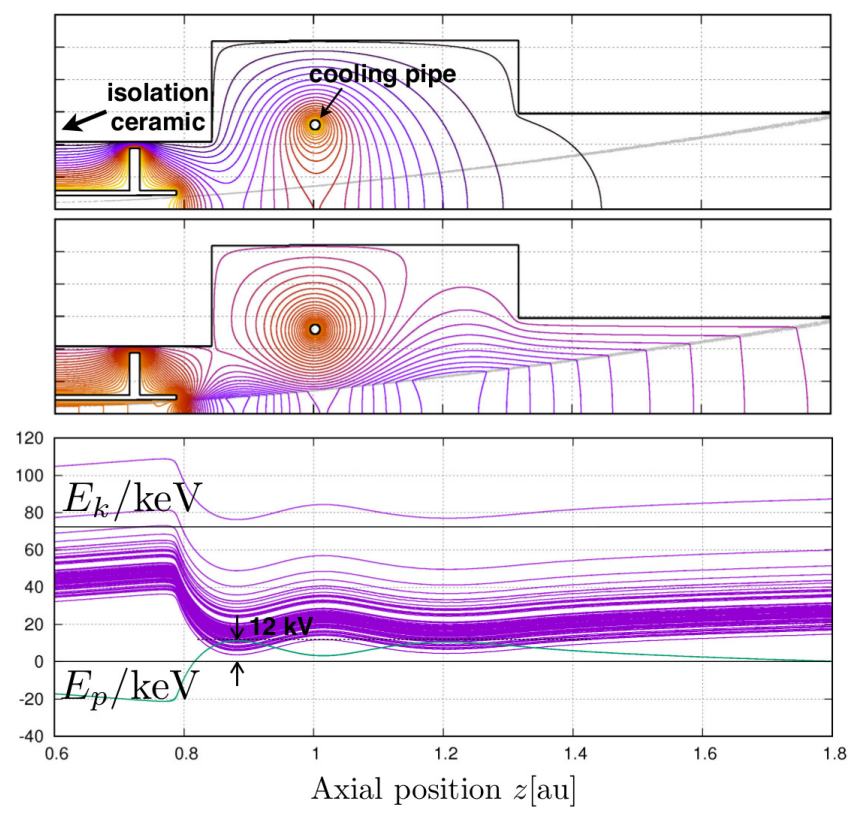

Fig. 5. The equipotential lines in the DS $\# 2$ for $I_{b}=0 \mathrm{~A}$ and $I_{b}=45 \mathrm{~A}$ are plotted at the top and middle respectively, while the potential and the kinetic energy for a large number of sample beam electrons as a function of the axial position are presented at the bottom.
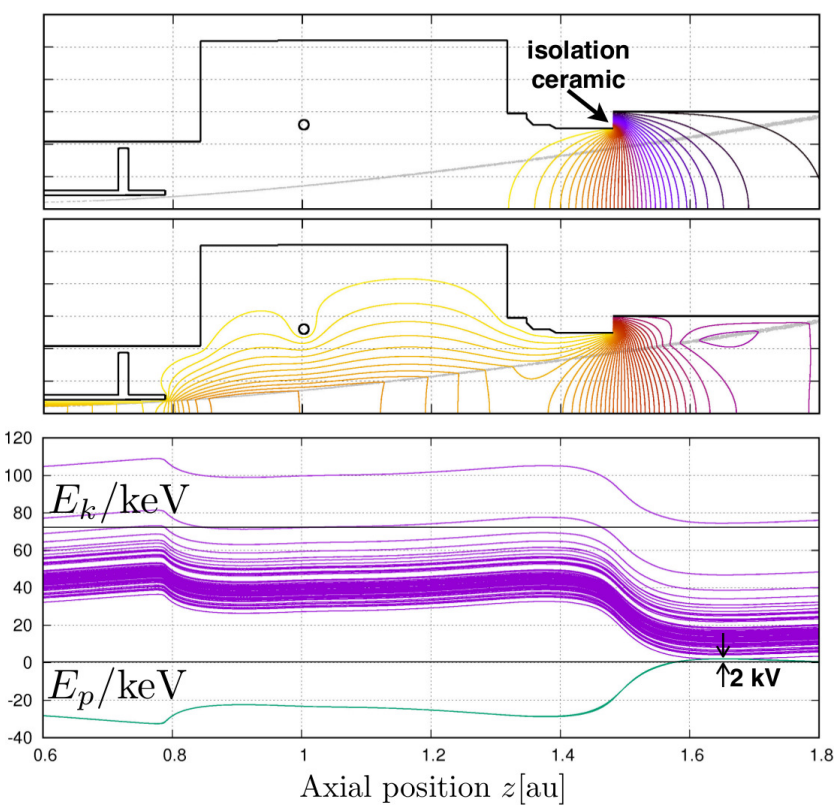

Fig. 6. The equipotential lines in the DS \#3 for $I_{b}=0 \mathrm{~A}$ and $I_{b}=45 \mathrm{~A}$ are plotted at the top and middle respectively, while the potential and the kinetic energy for a large number of sample beam electrons as a function of the axial position are presented at the bottom. 

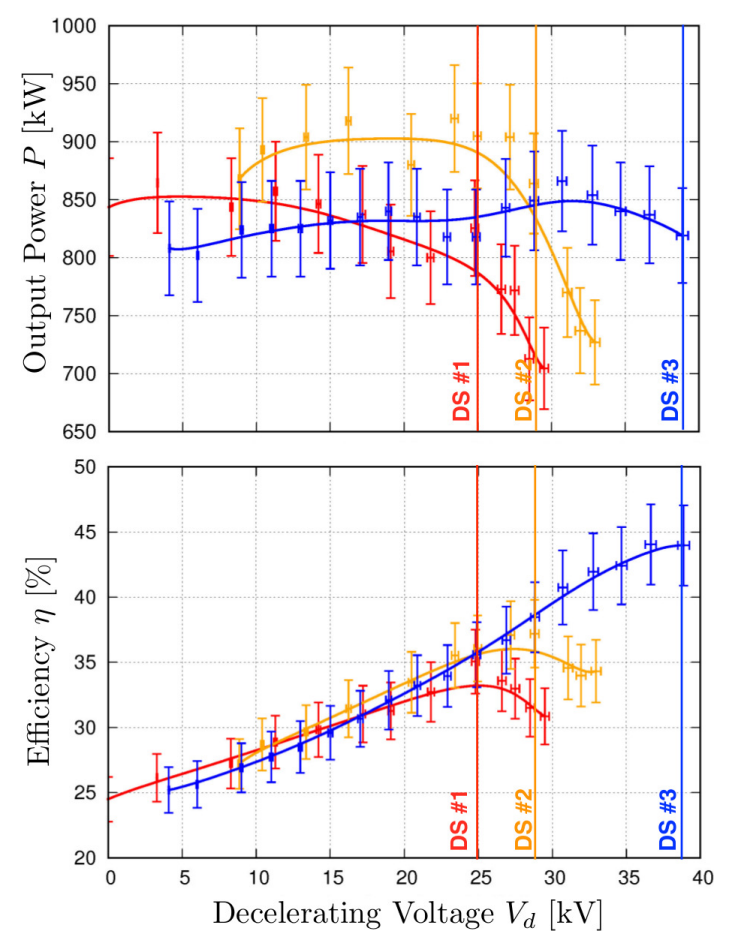

Fig. 7. Experimental comparison of output power (top) and overall efficiency (bottom) of the three different deceleration schemes.

isolation ceramic is placed at the bottom of the collector. In this way it was possible to test the approach in which the deceleration takes place at the bottom of the collector as shown in Fig. 6. A short circuit between the mirror box and the body structure was applied while an isolation ring replaced the metallic ring between the magnet and the gyrotron in order to isolate the grounded magnet from the mirror box on which a high voltage is applied. As it was expected, the applied retarding voltage and the gyrotron efficiency were significantly increased to $34 \mathrm{kV}$ and $53 \%$, respectively. A small deceleration takes place at the entrance of the beam in the mirror box only due to the space charge voltage depression and the final deceleration takes place at the collector entrance mainly due to the applied decelerating voltage.

The three deceleration schemes also have been experimentally tested. The experimental results, shown in Fig. 7, are qualitatively in agreement with the theoretical expectations. The optimal decelerating voltages were measured as $25 \mathrm{kV}$, $28 \mathrm{kV}$, and $38 \mathrm{kV}$ for DS \#1, DS \#2 and DS \#3, respectively. For each decelerating scheme, the power is not significantly influenced for voltages smaller than the optimal decelerating voltage, while for higher values it is obviously decreased. Exception is the case DS \#3, in which the limitation of $38 \mathrm{kV}$ is related to the maximum possible applied voltage of the power supply used in the experiments and not to problems related to the reflected electrons. The overall gyrotron efficiency which was experimentally measured was $35 \%, 37 \%$ and $44 \%$ for DS \#1, DS \#2 and DS \#3, respectively. The significant quantitative difference in the efficiency between the experimental and the theoretical results is related to the fact than no the optimal accelerating voltage and beam current
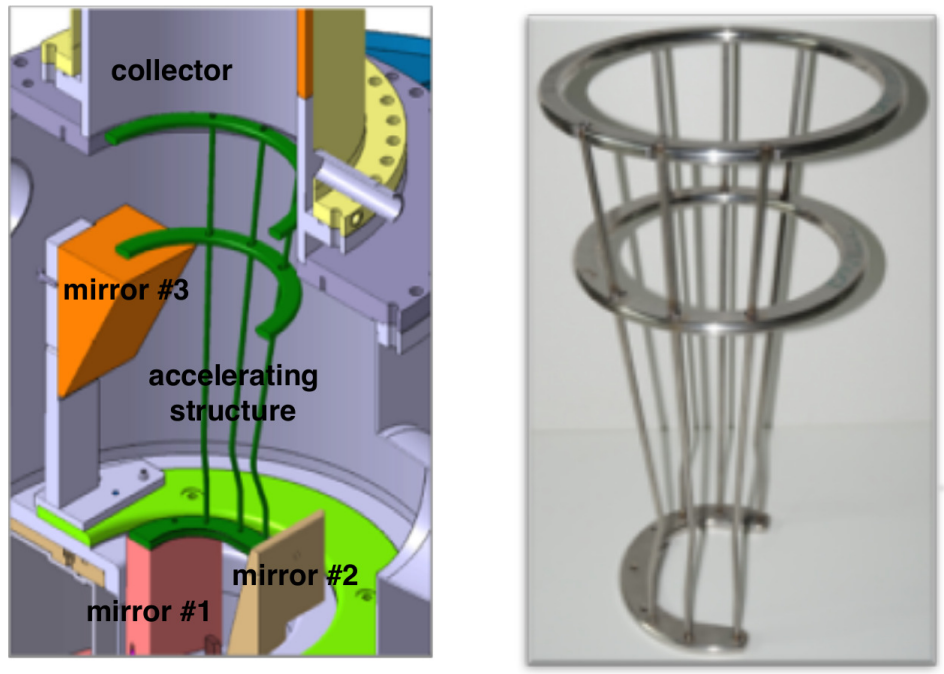

Fig. 8. 3D drawing of the accelerating structure installed in the SP prototype mirror box is presented at the left, while a photo of the accelerating structure is shown at the right.
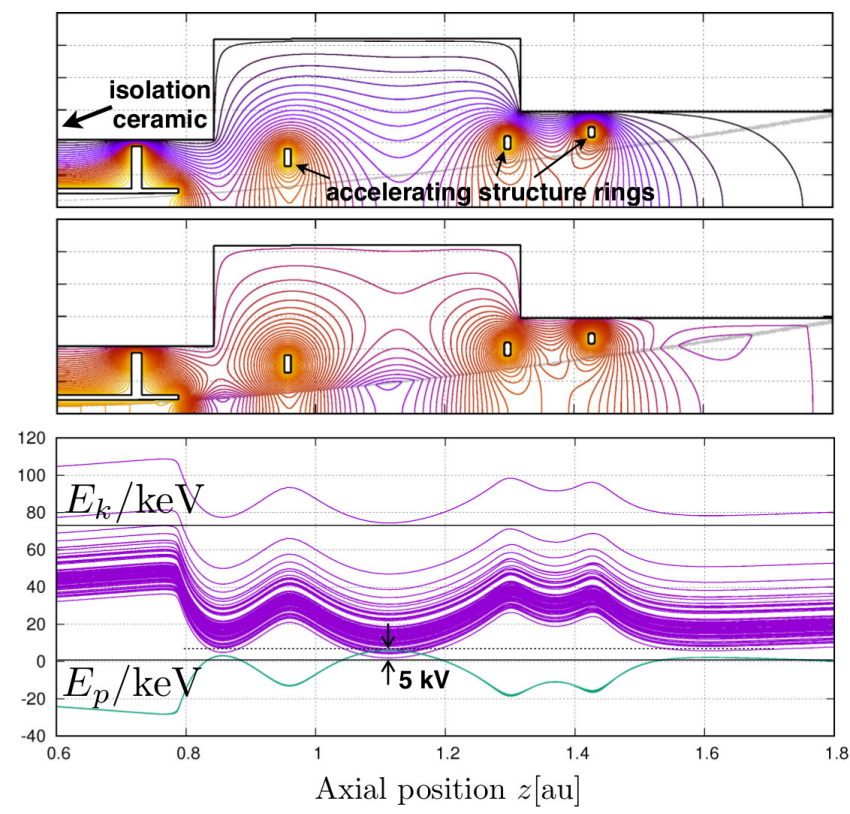

Fig. 9. The equipotential lines in the DS \#4 for $I_{b}=0 \mathrm{~A}$ and $I_{b}=45 \mathrm{~A}$ are plotted at the top and middle respectively, while the potential and the kinetic energy for a large number of sample beam electrons as a function of the axial position are presented at the bottom.

were used in the experiments.

\section{AcCELERAting Structure}

The main outcomes of the numerical and experimental investigation of the three deceleration schemes presented in Sec. II are that the overall efficiency of the gyrotron was significantly better in DS \#3, in which the deceleration takes place during the entrance of the beam in the collector, while it was possible to influence the efficiency by the presence of the dummy pipes (at DS \#2), which emulate the cooling pipes of the industrial prototype. Based on these observations, 
TABLE I

COMPARISON OF THE DECELERATING VOLTAGES AND GYROTRON EFFICIENCIES OF THE FOUR DIFFERENT DECELERATING SCHEMES.

\begin{tabular}{lcc}
\hline Deceleration Scheme & Voltage $V_{d}$ & Efficiency $\eta$ \\
\hline \#1 (Nominal) & $14 \mathrm{kV}$ & $36 \%$ \\
\#2 (CW prototype) & $23 \mathrm{kV}$ & $42 \%$ \\
\#3 (Ceramic on top) & $34 \mathrm{kV}$ & $53 \%$ \\
\#4 (Accelerating structure ) & $30 \mathrm{kV}$ & $49 \%$ \\
\hline
\end{tabular}

an effort took place to design an internal structure with the goal to increase the efficiency of the SP prototype keeping the isolation ceramic at the lower part of the gyrotron.

Such a structure has been designed and it is shown at the left of Fig. 8. The aim of this structure is to keep the kinetic energy of the beam electrons at a high level until the collector entrance. It consists of three rings at three different axial positions. It is fixed on top of the mirror \#1 which is at body voltage potential. Special attention took place to avoid any intersection of the geometry of the structure with the RF beam and to keep a safe distance between the rings and the electron beam. As is shown in Fig. 9, the presence of the rings in several axial positions keeps the influence of the beam space charge at low levels. In the worst case scenario concerning the azimuthal angle used in the axisymmetric simulation and applying the strict criterion of the slowest electron, the estimated retarding voltage is $30 \mathrm{kV}$ and the overall gyrotron efficiency is $49 \%$.

A comparison of the decelerating voltages and the efficiency of the four different decelerating schemes is presented in Table I. It shows that even if the gyrotron performance with DS \#4 is not as efficient as with DS \#3, where the isolation ceramic is installed at the bottom of the collector, it is significantly improved in comparison with DS \#1 and DS \#2. Such a structure has been manufactured as shown in Fig. 8 (rignt). The construction of the acceleration structure is quite simple due to the fact that no cooling is needed for SP operation. The experimental tests of the SP prototype gyrotron using this structure are planned for the following months.

\section{CONCLUSiON}

Three different schemes were theoretically studied for the deceleration of the spent electron beam of the SP prototype gyrotron. The results of this analysis are qualitatively in agreement with the results of the experiments. The results indicate that the most efficient scheme is when the beam deceleration takes place at the entrance of the collector.

However, the installation of the isolation ceramic at the bottom of the collector causes several technological difficulties correlated with the robustness of the ceramic and the high voltage which must be applied to the mirror box. In order to overcome these issues, the deceleration of the beam at the collector entrance is achieved using an acceleration structure at body potential keeping the isolation ceramic at the bottom part of the gyrotron. It should be noted that the design of an acceleration structure dedicated for a $\mathrm{CW}$ tube would be more complicated due to the fact that cooling will be necessary due to the presence of the stray radiation in the mirror box.
The idea of the acceleration structure could be taken into account for a possible upgrade of the industrial tube of the $170 \mathrm{GHz}, 1 \mathrm{MW}$, EU gyrotron for ITER. The major advantage of this idea is that no important technological modification of the gyrotron design is required while a significant gain of the overall efficiency is expected to be achieved.

\section{REFERENCES}

[1] K. Sakamoto, M. Tsuneoka, A. Kasugai, T. Imai, T. Kariya, K. Hayashi, and Y. Mitsunaka, "Major improvement of gyrotron efficiency with beam energy recovery", Physical review letters, vol. 73, no. 26, p. 3532, 1994.

[2] B. Piosczyk, C. T. Iatrou, D. Guenter, and T. Manfred, "Single-stage depressed collectors for gyrotrons", IEEE Transactions on Plasma Science, vol. 24, no. 3, pp. 579-585, June 1996. [Online]. Available: http://ieeexplore.ieee.org/document/532940/

[3] A. L. Goldenberg, V. N. Manuilov, M. A. Moiseev, and N. A. Zavolsky, "Energy spectra of electrons and depressed potential collector in gyrotrons", International Journal of Infrared and Millimeter Waves, vol. 18, no. 1, p. 43, Jan. 1997. [Online]. Available: http://dx.doi.org/10.1007/BF02677896

[4] G. Dammertz, S. Alberti, A. Arnold, E. Borie, V. Erckmann, G. Gantenbein, E. Giguet, R. Heidinger, J. P. Hogge, S. Illy, W. Kasparek, K. Koppenburg, M. Kuntze, H. P. Laqua, G. LeCloarec, Y. LeGoff, W. Leonhardt, C. Lievin, R. Magne, G. Michel, G. Muller, G. Neffe, B. Piosczyk, M. Schmid, K. Schworer, M. K. Thumm, and M. Q. Tran, "Development of a 140-GHz 1-MW continuous wave gyrotron for the W7-X stellarator", IEEE Transactions on Plasma Science, vol. 30, no. 3, pp. 808-818, Jun. 2002.

[5] J. Jelonnek, F. Albajar, S. Alberti, K. Avramidis, P. Benin, T. Bonicelli, F. Cismondi, V. Erckmann, G. Gantenbein, K. Hesch, J. P. Hogge, S. Illy, Z. C. Ioannidis, J. Jin, H. Laqua, G. P. Latsas, F. Legrand, G. Michel, I. G. Pagonakis, B. Piosczyk, Y. Rozier, T. Rzesnicki, I. G. Tigelis, M. Thumm, M. Q. Tran, and J. L. Vomvoridis, "From series production of gyrotrons for W7-X toward EU-1 MW gyrotrons for iter", IEEE Transactions on Plasma Science, vol. 42, no. 5, pp. 1135-1144, May 2014.

[6] I. G. Pagonakis, G. Gantenbein, J. Jelonnek, J. Jin, S. Illy, S. Kern, B. Piosczyk, T. Rzesnicki, M. Thumm, K. A. Avramides, J. L. Vomvoridis, O. Dumbrajs, P. Benin, Y. Rozier, S. Alberti, J. P. Hogge, C. Schlatter, M. Q. Tran, A. Bruschi, M. Lontano, Z. C. Ioannidis, G. P. Latsas, I. G. Tigelis, F. Albajar, T. Bonicelli, and F. Cismondi, "Design of the EU-1MW gyrotron for ITER", in Proc. IEEE 14th Int. Vacuum Electronics Conf. (IVEC), May 2013, pp. 1-2.

[7] A. Litvak, G. Denisov, M. Agapova, V. Myasnikov, L. Popov, E. Tai, S. Usachev, V. Zapevalov, A. Chirkov, V. Ilin et al., "Development in Russia of $170 \mathrm{GHz}$ gyrotron for ITER", in Infrared, Millimeter and Terahertz Waves (IRMMW-THz), 2011 36th International Conference on. IEEE, 2011, pp. 1-3.

[8] V. Zapevalov, G. Denisov, V. Flyagin, A. S. Fix, A. Kuftin, A. Litvak, M. Agapova, V. Iljin, V. Khmara, V. Myasnikov et al., "Development of $170 \mathrm{GHz} / 1 \mathrm{MW}$ Russian gyrotron for ITER", Fusion Engineering and Design, vol. 53, no. 1, pp. 377-385, 2001.

[9] J.-P. Hogge, T. Goodman, S. Alberti, F. Albajar, K. Avramides, P. Benin, S. Bethuys, W. Bin, T. Bonicelli, A. Bruschi et al., "First experimental results from the European union 2-MW coaxial cavity iter gyrotron prototype", Fusion Science and Technology, vol. 55, no. 2, pp. 204212, 2009.

[10] T. Rzesnicki, G. Gantenbein, J. Jelonnek, J. Jin, I. G. Pagonakis, B. Piosczyk, A. Samartsev, A. Schlaich, and M. Thumm, "2 MW, $170 \mathrm{GHz}$ coaxial-cavity short-pulse gyrotron — single stage depressed collector operation," in Proc. and Terahertz waves (IRMMW-THz) 2014 39th Int. Conf. Infrared, Millimeter, Sep. 2014, pp. 1-2.

[11] I. G. Pagonakis, F. Albajar, S. Alberti, K. Avramidis, T. Bonicelli, F. Braunmueller, A. Bruschi, I. Chelis, F. Cismondi, G. Gantenbein et al., "Status of the development of the EU $170 \mathrm{GHz} / 1 \mathrm{MW} / \mathrm{CW}$ gyrotron", Fusion Engineering and Design, vol. 96, pp. 149-154, 2015.

[12] Z. C. Ioannidis, T. Rzesnicki, F. Albajar, S. Alberti, K. A. Avramidis, W. Bin, T. Bonicelli, A. Bruschi, I. Chelis, P. E. Frigot, G. Gantenbein, V. Hermann, J. P. Hogge, S. Illy, J. Jin, J. Jelonnek, W. Kasparek, G. Latsas, C. Lechte, F. Legrand, T. Kobarg, I. G. Pagonakis, Y. Rozier, C. Schlatter, M. Schmid, I. G. Tigelis, M. Thumm, M. Q. Tran, A. Zein, and A. Zisis, "CW experiments with the EU 1-MW, 170-GHz industrial prototype gyrotron for ITER at KIT," IEEE Transactions on Electron Devices, vol. 64, no. 9, pp. 3885-3892, Sep. 2017. 
[13] I. G. Pagonakis, K. Avramides, S. Illy, P. Kalaria, G. Gantenbein, and J. Jelonnek, "Electron beam simulation in the overall gyrotron geometry", in 9th Int. Workshop Strong Microwaves and Terahertz Waves: Sources and Applications, Nizhny Novgorod, Russia, 24-30 July 2014, 2014

[14] J. G. Pagonakis and J. L. Vomvoridis, "The self-consistent 3D trajectory electrostatic code ARIADNE for gyrotron beam tunnel simulation", in Proc. Infrared and Millimeter Waves, Conf. Digest of the 2004 Joint 29th Int. Conf. 2004 and 12th Int. Conf. Terahertz Electronics, Sep. 2004, pp. 657-658.

[15] K. A. Avramides, I. G. Pagonakis, C. T. Iatrou, and J. L. Vomvoridis, "EURIDICE: A code-package for gyrotron interaction simulations and cavity design", in EPJ Web of Conferences, vol. 32. EDP Sciences, 2012, p. 04016

Ioannis Gr. Pagonakis received the B.S. degree in physics from the University of Crete, Crete, Greece, in 1997, and the Ph.D. degree from the School of Electrical and Computer Engineering, National Technical University of Athens, Greece, in 2005. Since 2010 he works as a researcher with the Karlsruhe Institute of Technology KIT. His research interests include the computer-aided design, dynamics of electron beams, the gun and multi-stage depressed collector design for gyrotrons.

Stefan Illy received the Dipl.-Phys. and Dr.rer.nat. degrees in physics from the Karlsruhe Institute of Technology (KIT), Karlsruhe, Germany, in 1993 and 1997, respectively. He has been with the High Power Microwave Technology Division, Institute for Pulsed Power and Microwave Technology, KIT, since 1999, where he currently leads the Gyrotron Interaction Analysis and Components Group.

Tomasz Rzesnicki received the Dipl.-Ing. degree in the electrical engineering from the Karlsruhe Institute of Technology (KIT), Karlsruhe, Germany, and Politechnika Gdanska, Gdanska, Poland, in 2002, and the Ph.D. degree from KIT in 2007. He has been with KIT since 2003, where he is currently involved in the development of high-power gyrotrons. He became a part of the Gyrotron Research Group as a Young Scientist.

Zisis C. Ioannidis received the B.S. degree in physics, the M.S. degree in electronics, and the Ph.D. degree from the National and Kapodistrian University of Athens, Athens, Greece, in 2002, 2004, and 2008, respectively. Since 2016, he has been with the Institute for Pulsed Power and Microwave Technology, Karlsruhe Institute of Technology, Karlsruhe, Germany.

Konstantinos A. Avramidis was born in Athens, Greece, in 1971. He received the Dipl.Ing. and Ph.D. degrees from the School of Electrical and Computer Engineering, National Technical University of Athens, in 1998 and 2006, respectively. He joined the Institute for Pulsed Power and Microwave Technology, Karlsruhe Institute for Technology, Karlsruhe, Germany, in 2013. Dr. Avramidis is a member of the Technical Chamber of Greece.

Gerd Gantenbein received the Dipl. Phys. and the Dr.-Ing. Degrees in electrical engineering from the University of Karlsruhe, Karlsruhe, Germany, in 1988 and 1993, respectively. Since 2005, he has been with the Institute for Pulsed Power and Microwave Technology of the Karlsruhe Institute of Technology, Karlsruhe, where he is the Head of the division High Power Microwave Technology.
Thorsten Kobarg received the Graduate Engineer degree in mechanical engineering with a focus in engineering design from the Beuth University of Applied Sciences, Berlin, Germany, in 2006. Since 2013, he has been in charge of mechanical design and the development of Gyrotron systems with the Institute for Pulsed Power and Microwave Technology, Karlsruhe Institute of Technology, Karlsruhe, Germany.

Bernhard Piosczyk received the Dipl.Ing. degree in physics from the Technical University of Berlin, Berlin, Germany, in 1969, and the Dr.rer.nat. degree from the University of Karlsruhe, Karlsruhe, Germany, in 1974. Since 1987, he has been involved in the development of high-power gyrotrons. Since 2008 , he has been retired and doing some consultancy.

Manfred Thumm (SM94-F02) received the Dipl.-Phys. and Dr.rer.nat. degrees in physics from the University of Tuebingen, Tuebingen, Germany, in 1972 and 1976, respectively. He was the Director of the Institute for Pulsed Power and Microwave Technology, Forschungszentrum Karlsruhe, Karlsruhe, Germany, from 1999 to 2011. His current research interests include the development of high-power gyrotrons, transmission lines, and antennas for nuclear fusion plasma heating.

John Jelonnek (M97-SM15) received the Dipl.-Ing. and the Dr.-Ing. degrees in electrical engineering from the Hamburg University of Technology, Hamburg, Germany, in 1992 and 2000, respectively. He has been a Professor and the Director of the Institute for Pulsed Power and Microwave Technology with the Karlsruhe Institute of Technology, Karlsruhe, Germany, since 2011. 